\title{
INNER-SHELL PHOTOIONIZATION STUDIES OF NEUTRAL ATOMIC NITROGEN
}

\author{
W. C. Stolte ${ }^{1,2,6}$, V. Jonauskas ${ }^{3}$, D. W. Lindle ${ }^{1,7}$, M. M. Sant'Anna ${ }^{4}$, and D. W. Savin ${ }^{5}$ \\ ${ }^{1}$ Department of Chemistry, University of Nevada, Las Vegas, NV 89154, USA \\ 2 Advanced Light Source, Lawrence Berkeley National Laboratory, Berkeley, CA 94720, USA \\ ${ }^{3}$ Institute of Theoretical Physics and Astronomy, Vilnius University, Goštauto St. 12, LT-01108 Vilnius, Lithuania \\ ${ }^{4}$ Instituto de Física, Universidade Federal do Rio de Janeiro, 21941-972 Rio de Janeiro, Brazil \\ ${ }^{5}$ Columbia Astrophysics Laboratory, Columbia University, New York, NY 10027, USA \\ Received 2014 December 18; accepted 2016 January 5; published 2016 February 17
}

\begin{abstract}
Inner-shell ionization of a $1 s$ electron by either photons or electrons is important for X-ray photoionized objects such as active galactic nuclei and electron-ionized sources such as supernova remnants. Modeling and interpreting observations of such objects requires accurate predictions for the charge state distribution (CSD), which results as the $1 s$-hole system stabilizes. Due to the complexity of the complete stabilization process, few modern calculations exist and the community currently relies on 40-year-old atomic data. Here, we present a combined experimental and theoretical study for inner-shell photoionization of neutral atomic nitrogen for photon energies of $403-475 \mathrm{eV}$. Results are reported for the total ion yield cross section, for the branching ratios for formation of $\mathrm{N}^{+}, \mathrm{N}^{2+}$, and $\mathrm{N}^{3+}$, and for the average charge state. We find significant differences when comparing to the data currently available to the astrophysics community. For example, while the branching ratio to $\mathrm{N}^{2+}$ is somewhat reduced, that for $\mathrm{N}^{+}$is greatly increased, and that to $\mathrm{N}^{3+}$, which was predicted to be zero, grows to $\approx 10 \%$ at the higher photon energies studied. This work demonstrates some of the shortcomings in the theoretical CSD data base for inner-shell ionization and points the way for the improvements needed to more reliably model the role of innershell ionization of cosmic plasmas.
\end{abstract}

Key words: atomic data - atomic processes - methods: laboratory: atomic - opacity

\section{INTRODUCTION}

Inner-shell ionization of a $1 s$ electron by either photons or electrons can be an important process for a range of cosmic sources. These include X-ray photoionized objects such as active galactic nuclei or X-ray binaries (Kallman \& Bautista 2001), photoionization of interstellar dust grains (Dwek \& Smith 1996), and shock-driven gas such as in supernova remnants (Vink 2012). The resulting $1 s$-hole system relaxes via a complicated cascade of fluorescence and/or Auger-ejected electrons. This process affects both the emitted spectrum of a source as well as the ionization structure of the gas.

Driven by the astrophysical importance of inner-shell ionization, Kaastra \& Mewe (1993) carried out the first, and to-date the only, comprehensive study into the resulting fluorescence and Auger yields for all ionization stages of the elements from beryllium to zinc. These data are derived from theoretical calculations published between 1969 and 1972 using $L S$-coupling and a central field approximation. The data are also presented using configuration-averaging, thereby providing no fine-structure information for the fluorescence or Auger processes.

Given the understandable limitations of the Kaastra \& Mewe (1993) data, in the past decade, there has been significant theoretical effort using relativistic $L S J$ methods to calculate the fluorescence yield due to an initial $1 s$ hole (e.g., Bautista et al. 2003; Gorczyca et al. 2003; Palmeri et al. 2003a, 2003b, 2008a, 2008b, 2011, 2012; Mendoza et al. 2004; García et al. 2005; Gorczyca et al. 2006; Hasoğlu et al. 2006; García et al. 2009). That work finds a number of differences with Kaastra \& Mewe (1993). For example, fluorescence channels that were

\footnotetext{
${ }^{6}$ Present address: National Security Technologies, LLC (NSTec), Livermore, CA 94550, USA.

7 Deceased, 2014 October.
}

thought to be closed are in fact open and channels that are open can have fluorescence yields that differ by factors of several or more.

There is far less theoretical work on the resulting charge state distribution (CSD). This is not surprising given the complexity of the complete stabilization process. Inner-shell ionization of iron, for example, can result in the ejection of up to eight additional electrons (Kallman \& Bautista 2001). However, this multiple Auger process is often ignored by astrophysicists, probably because the needed state-of-the-art data are lacking. This omission likely hinders our astrophysical understanding of cosmic sources as multiple electron emission can dramatically change the predicted ionization structure of an astrophysical source, particularly in ionizing plasmas (Hahn \& Savin 2015a, 2015b).

To begin to address this matter, here we report a combined theoretical and experimental study into inner-shell photoionization on neutral atomic nitrogen. The rest of this paper is organized as follows. In Section 2, we briefly review the theoretical method used. The experimental method is presented in Section 3. The theoretical and experimental results are presented and discussed in Section 4. Lastly, we give a summary of our findings in Section 5.

\section{THEORETICAL APPROACH}

Our photoionization calculations account for both the promotion of a $1 s$ electron into the continuum and also the resonant photoexcitation of a $1 s$ electron. The former leads directly to ionization, but both processes contribute to the total ionization cross section through a sequential cascade of radiative and Auger transitions as the system relaxes. In addition, we account for direct ionization of an electron from higher shells. In our previous investigation (Sant'Anna 
et al. 2011), we determined the resonance structure in the 390-420 eV range using an R-matrix approach. Our aim here, though, is to study the formation of ions due to Auger cascades.

We have calculated energy levels, radiative and Auger transition probabilities, and photoionization cross sections using the Flexible Atomic Code (FAC), which employs the Dirac-Fock-Slater method (Gu 2008). For photoexcitations from the ground level to the $1 s 2 s^{2} 2 p^{3}\left({ }^{3} \mathrm{D}\right) 3 p\left({ }^{4} \mathrm{P}\right)$, $1 s 2 s^{2} 2 p^{3}\left({ }^{3} \mathrm{D}\right) 4 p\left({ }^{4} \mathrm{P}\right)$, and $1 s 2 s^{2} 2 p^{3}\left({ }^{3} \mathrm{D}\right) 5 p\left({ }^{4} \mathrm{P}\right)$ terms, experimentally measured energies were used. However, we use our calculated oscillator strengths for these transitions, which correspond to the strongest ones in the energy range considered. The total number of levels included is 3781 . We used 1787 levels for $\mathrm{N}, 1470$ for $\mathrm{N}^{+}, 480$ for $\mathrm{N}^{2+}$, and 44 for $\mathrm{N}^{3+}$. The necessary bound and continuum wavefunctions for each ion were calculated using the local central potential for the ground configuration. The wavefunctions are obtained separately for each charge state using self-consistent field procedures. As a result, the wavefunctions for different charge states are not orthogonal. The calculated photoionization cross sections and Auger transition rates involve using wavefunctions from different charge states. The non-orthogonality of the wavefunctions for the different ionization states is expected to have only a small influence on these calculations. When calculating the relevant matrix elements, the additional terms introduced are small, often have different signs, and approximately cancel out when summed (Cowan 1981). This is sometimes referred to as "compensation effects." The mixing of all configurations belonging to the same ionization stage is taken into account.

Level populations have been estimated in every step of the cascade process using (Palaudoux et al. 2010; Jonauskas et al. 2011)

$$
N_{j}=\sum_{i} N_{i} \frac{A_{i j}}{A_{i}}
$$

where $N_{j}$ is the population of level $j, N_{i}$ is the population of the initial level $i, A_{i j}$ is the Auger transition probability from $i$ to $j$, and $A_{i}$ is the total decay rate of $i$, from both Auger and radiative transitions. The initial level populations due to photoionization and photoexcitation at a given photon energy have been obtained from the corresponding cross sections divided by the total cross section summing all processes at that energy.

Double photoionization (DPH) is treated here as a sequence of two independent processes. It begins with the photoionization of a single electron. This photoelectron then causes electron-impact ionization of the remaining system. A similar approach has been successfully applied to the investigation of the electron-impact double-ionization process (Jonauskas et al. 2014). Within this formalism, the DPH cross section from level $i$ to $f$ can be expressed as

$$
\sigma_{i f}^{\mathrm{DPH}}\left(\varepsilon_{\mathrm{ph}}\right)=\sum_{j} \sigma_{i j}^{\mathrm{PH}}\left(\varepsilon_{\mathrm{ph}}\right) p_{j}\left(\varepsilon_{\mathrm{ph}}\right) \frac{\sigma_{j f}^{\mathrm{CI}}\left(\varepsilon_{1}\right)\left(N_{n l}\right)^{2 / 3}}{4 \pi \bar{R}_{n l}^{2}} .
$$

Here, $\varepsilon_{\mathrm{ph}}$ is the energy of the photon and $\varepsilon_{1}$ is the energy of the photoelectron, which ionizes an electron from the $n l$ shell. The population of level $j$, produced by the photoionization process from level $i$, is given by $p_{j}$. The factor $\sigma_{j f}^{\text {CI }}\left(\varepsilon_{1}\right)\left(N_{n l}\right)^{2 / 3} / 4 \pi \bar{R}_{n l}^{2}$ determines the probability of electron-impact ionization (Gryziński 1965), where $\sigma_{j f}^{\mathrm{CI}}\left(\varepsilon_{1}\right)$ is the electron-impact ionization cross section from level $j$ to $f, N_{n l}$ is the number of electrons in the $n l$ shell, and $\bar{R}_{n l}$ is the mean distance of the electrons from the nucleus.

We also investigated the importance of ionization due to the sudden change of the ion charge after photoionization, a process known as shake-off. For this, we used the sudden perturbation approximation (Jonauskas et al. 2009), which is expected to be valid for high energy photons when the resulting initial photoelectron leaves the system at high velocity and does not have time to interact with the remaining bound electrons. However, these calculations strongly overestimate the yield of the $\mathrm{N}^{3+}$ ions, indicating that the approach was not valid at the energies investigation, and hence the results were not included in our final calculations.

\section{EXPERIMENTAL METHOD}

The measurements were performed on the undulator beamline 8.0.1.3 at the Advanced Light Source located at the Lawrence Berkeley National Laboratory, in Berkeley, CA, USA. The basic experimental setup has been previously described in Stolte et al. (2008), as has the method used to generate atomic species (Sant'Anna et al. 2011), which is similar to that used for the creation of atomic chlorine (Stolte et al. 2013) and atomic oxygen (Stolte et al. 1997; McLaughlin et al. 2013).

In short, atomic $\mathrm{N}$ was generated by flowing commercially obtained $99.9995 \%$ pure gaseous $\mathrm{N}_{2}$ through a microwave discharge cavity. The products of this nitrogen plasma were a mixture of atoms and molecules in ground and excited states. A combination of a fast gas flow rate with teflon and phosphorus pentoxide coatings on the flow tubes, in addition to tubing shape and discharge distance, reduced wall recombination effects and strongly quenched nitrogen atoms created in the long-lived $1 s^{2} 2 s^{2} 2 p^{3}\left({ }^{2} \mathrm{D}^{\circ},{ }^{2} \mathrm{P}^{\circ}\right)$ metastable states. A final enhancement was the addition of a constant magnetic field, satisfying the electron-cyclotron resonance condition, being perpendicularly superimposed on the the $2.45 \mathrm{GHz}$ electric field of the microwave cavity. The resulting mixture of $1 s^{2} 2 s^{2} 2 p^{3}\left({ }^{4} \mathrm{~S}^{\circ}\right)$ ground-state nitrogen atoms and molecular $\mathrm{N}_{2}$ were constrained to flow through a small orifice of $0.5 \mathrm{~mm}$ diameter before entering the interaction region to interact with the X-rays.

Partial ion yields were measured using the method described in the references above and includes a mixture of contributions from both ground-state atomic $\mathrm{N}$ and molecular $\mathrm{N}_{2}$. Although we eventually subtract it out, the presence of a molecular signal within our spectra provides an excellent internal energy calibration (Chen et al. 1989; Semenov et al. 2006). The cross section, $\sigma^{q+}(E)$, as a function of photon energy, $E$, for photoionization of atomic nitrogen to an ion of charge $q+$ can be obtained from (Samson \& Pareek 1985)

$$
\sigma^{q+}(E)=C_{q^{+}}\left(I_{\mathrm{on}}^{q+}-f I_{\mathrm{off}}^{q+}\right),
$$

where $I_{\text {on }}^{q+}$ and $I_{\text {off }}^{q+}$ are normalized ion yields measured as a function of photon energy with the microwave discharge on or off, respectively. $C_{q+}$ is a constant dependent on the number density of nitrogen atoms and the ion-collection efficiency of the apparatus. Absolute data for single and multiple photoionization of $\mathrm{N}_{2}$ from Stolte et al. (1998) were used to determine values for the constants $C_{q+}$. The parameter $f=\rho\left(\mathrm{N}_{2}^{\text {on }}\right) / \rho\left(\mathrm{N}_{2}^{\text {off }}\right)$ represents the fraction of $\mathrm{N}_{2}$ molecules, 


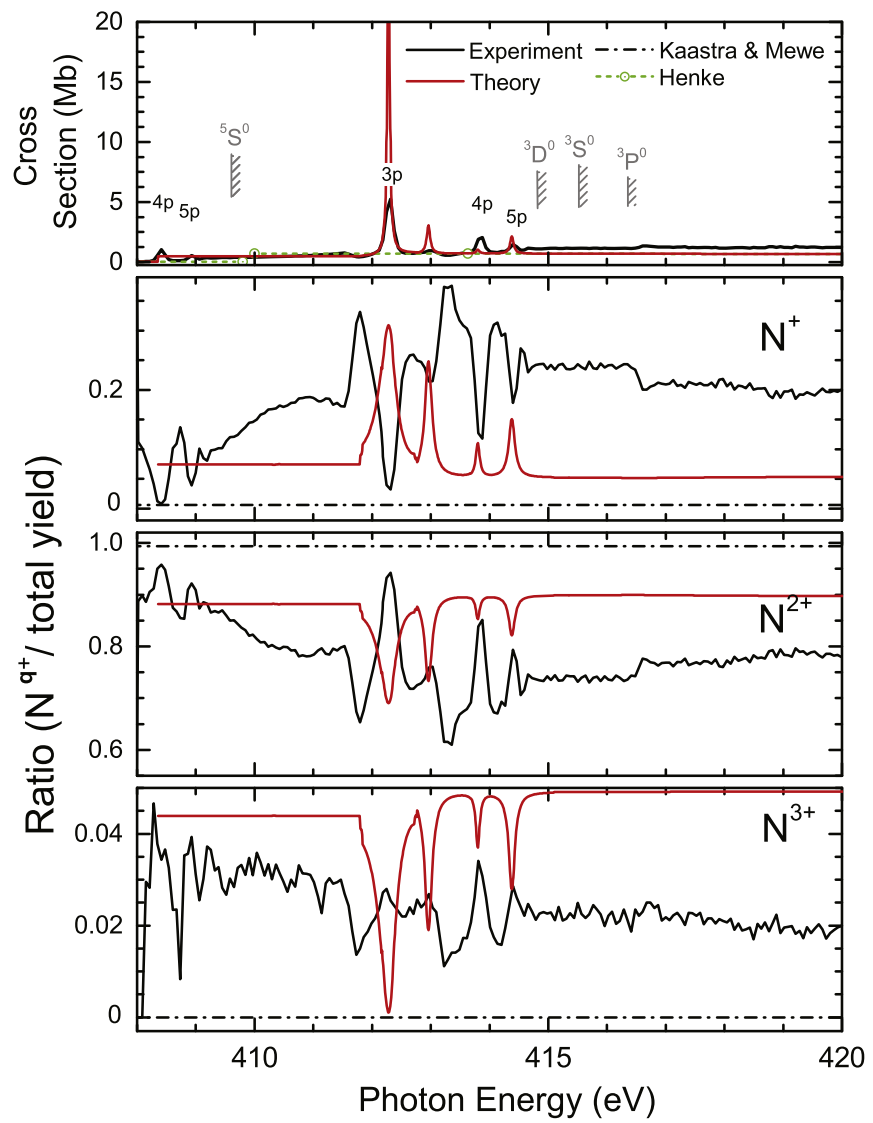

Figure 1. Top panel shows the photoionization cross section for total ion yield (TIY) of atomic $\mathrm{N}$ near the $1 s$-ionization threshold. Various core excitations and associated autoionizing resonances are labeled. Our experimental results are shown in black (with an estimated uncertainty of $12 \%$ in magnitude and $\sim 50 \mathrm{meV}$ in energy). Our theoretical results are in red. Also shown are the measurements of Henke et al. (1993) in green. The corresponding branching ratios for forming $\mathrm{N}^{q+}$ relative to the TIY are shown in the lower three panels. The published theoretical results of Kaastra \& Mewe (1993) are shown by the dot-dashed lines.

which do not dissociate in the discharge, with $\rho\left(\mathrm{N}_{2}^{\text {on }}\right)$ and $\rho\left(\mathrm{N}_{2}^{\text {off }}\right)$ being number densities of $\mathrm{N}_{2}$ with the microwave discharge on or off, respectively. The value of $f$ is empirically chosen to eliminate the molecular peaks from the measured ion yields via a weighted subtraction (Samson et al. 1986; Samson \& Angel 1990; Stolte et al. 1997). As noted above, the dissociation fraction is $1-f$, or approximately $4 \%$ here. Finally, the collection efficiency for each ion $\mathrm{N}^{q+}$ produced by photoionization of atomic nitrogen was assumed to be equal to the collection efficiency of the same $\mathrm{N}^{q+}$ generated by dissociative photoionization of $\mathrm{N}_{2}$.

\section{RESULTS AND DISCUSSION}

In Figure 1, we present our measured and theoretical cross sections for the total ionization yield (TIY) between $408-420 \mathrm{eV}$, which is near the the 1 -ionization threshold. Using our experimental and theoretical results, we have computed the branching ratio for forming $\mathrm{N}^{q+}$ relative to the total atomic ion yield. Figure 2 shows our results over the larger energy range of 410-475 eV. Also shown in these figures are the experimental cross section results of Henke et al. (1993) and the theoretical branching ratio results of Kaastra \& Mewe

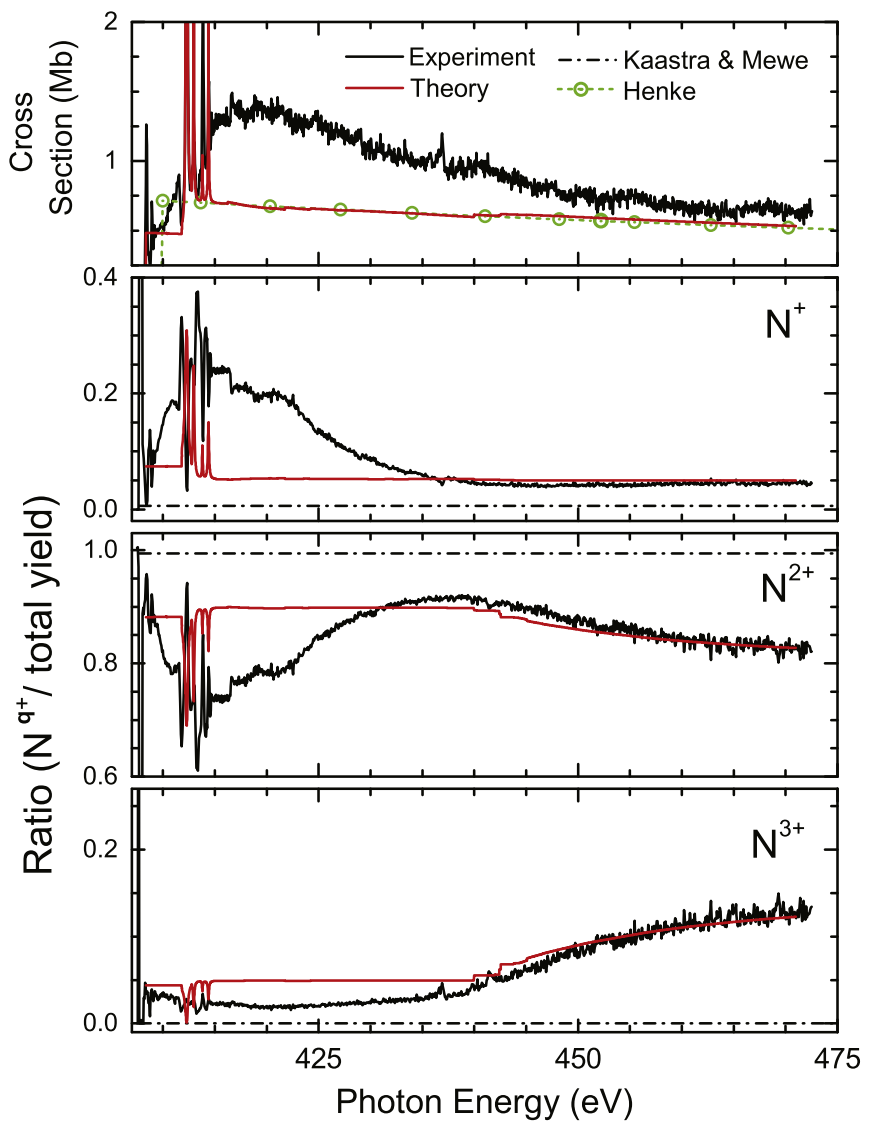

Figure 2. Same as Figure 1, but from 410 to $475 \mathrm{eV}$.

Table 1

Present Experimental and Theoretical Total Photoionization Cross Section of Atomic N Along with the Published Results of Henke et al. (1993)

\begin{tabular}{lccc}
\hline \hline Photon Energy $(\mathrm{eV})$ & \multicolumn{3}{c}{ Cross Section $(\mathrm{Mb})$} \\
\cline { 2 - 4 } & Experiment & Theory & Henke et al. \\
\hline 409.80 & 0.539 & 0.481 & 0.027 \\
410.00 & 0.531 & 0.481 & 0.714 \\
413.64 & 0.905 & 0.723 & 0.700 \\
420.33 & 1.35 & 0.660 & 0.674 \\
427.12 & 1.20 & 0.646 & 0.650 \\
434.03 & 1.00 & 0.624 & 0.626 \\
441.05 & 0.955 & 0.610 & 0.603 \\
448.19 & 0.775 & 0.604 & 0.581 \\
452.20 & 0.712 & 0.590 & 0.569 \\
455.44 & 0.748 & 0.580 & 0.560 \\
462.80 & 0.648 & 0.557 & 0.540 \\
470.29 & 0.637 & 0.534 & 0.520 \\
\hline
\end{tabular}

Note. The cross sections are given in units of megabarns $(\mathrm{Mb})$, which correspond to $10^{-18} \mathrm{~cm}^{2}$. Our estimated combined statistical and systematic uncertainty on the experimental results is $12 \%$ for the cross section and $\approx 50 \mathrm{meV}$ for the photon energy.

(1993). In Table 1, we present our experimental and theoretical total photoionization cross sections along with the experimental results of Henke et al. (1993). Table 2 presents the branching ratio data at selected photon energies, along with the average final charge state, which is also shown in Figure 3.

Comparing our experimental TIY cross section data to that of Henke et al. (1993), we find a significant difference between the two. This should not be surprising since the previous results 
Table 2

Our Experimental and Theoretical Branching Ratios for Formation of $\mathrm{N}^{+}, \mathrm{N}^{2+}$, and $\mathrm{N}^{3+}$ ions, Following Photoionization of Atomic N near the $1 s$ Ionization Threshold

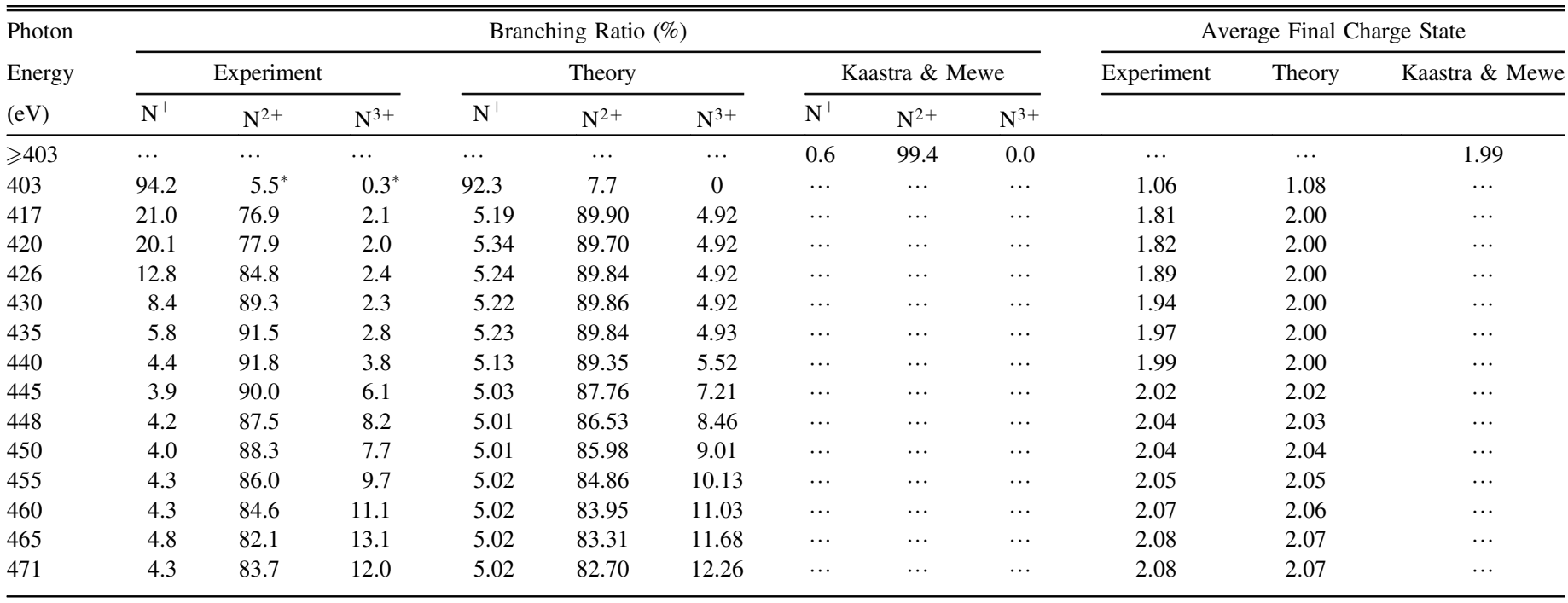

Note. The estimated combined statistical error and systematic error on the experimental results is $3 \%$, and approximately 50 meV for the photon energy, except for the starred data where the estimated error is 50\%. The theoretical values of Kaastra \& Mewe (1993) are included for comparison.

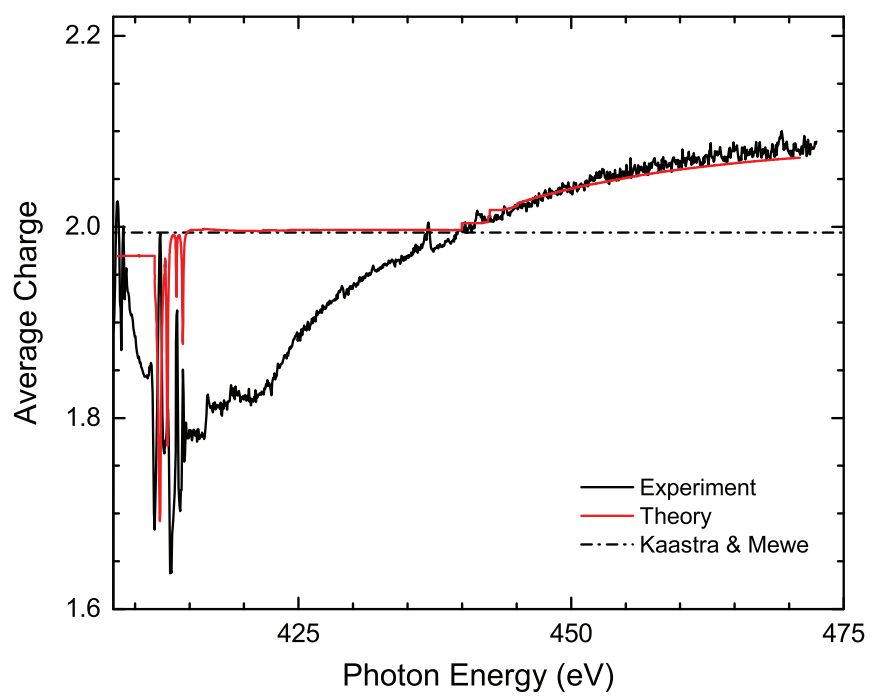

Figure 3. Average nitrogen charge state following the photoionization of atomic nitrogen. The black curve presents the experimental data, the red curve represents our present theoretical results, and the dot-dashed line shows the published theoretical results of Kaastra \& Mewe (1993).

were calculated from molecular nitrogen data taken at fixed photon energies using lamp sources and then dividing those absolute cross sections by two. This estimate of dividing the molecular value by two should become valid with high energy, but, as can be seen in Figure 2, it is still nearly 20\% lower at $55 \mathrm{eV}$ above threshold. We also note that only one actual measured point at $452.2 \mathrm{eV}$ from Henke et al. (1993) lies within our energy region. The rest are calculated results using dispersion equations, empirical relations to directly relate the incident photon energy with an atomic photoabsorption cross section. Also, as a result of the coarse energy grid used by Henke et al. (1993), none of the resonance structure was observed at the time.

We find a rich resonance structure in our data. In the $408-415 \mathrm{eV}$ range, the measured cross section shows several clear resonances due to core-excited autoionizing $1 s 2 s^{2} 2 p^{3} n p$ resonances $(n=3-5)$, leading up to the various series limits for the core excitation (Sant'Anna et al. 2011). These show up in the branching ratios as dips in the $\mathrm{N}^{+}$branching ratio, whereas they tend to appear as peaks in the $\mathrm{N}^{2+}$ and $\mathrm{N}^{3+}$ ratios. Interestingly, the branching ratios show an even greater variation than is seen in the cross section. This rapidly varying behavior continues until the photon energy converges to the ${ }^{3} \mathrm{D}^{\circ},{ }^{3} \mathrm{~S}^{\circ}$, and ${ }^{3} \mathrm{P}^{\circ}$ series limits for the core excitation, above which the behavior is smoother. Some of this rapidly varying behavior may be due to the measurement method, as is discussed in Sant'Anna et al. (2011). The experiment is performed by measuring spectra with the microwave discharge on and off and subtracting results. The residual component of the $\mathrm{N}_{2}$ contribution with the discharge on is not exactly the same as the room temperature $\mathrm{N}_{2}$ contribution obtained with microwave discharge off, since vibrationally excited states may show different signatures. This, and statistical limitations, may cause part of the discrepancy seen between experiment and theory.

We also note that the resonances above the $1 s$-ionization threshold, the ${ }^{5} \mathrm{~S}^{\circ}$ limit at $409.64 \mathrm{eV}$, show clear Fano-Beutler profiles. This indicates that there are strong interferences between the core-excited intermediate resonance states and the open continua. These profiles show up as valleys in the $\mathrm{N}^{+}$ ratio and as peaks in the $\mathrm{N}^{2+}$ and $\mathrm{N}^{3+}$ ratios.

Our calculated first ionization threshold energy is $407.36 \mathrm{eV}$, which is $2.28 \mathrm{eV}$ smaller than the experimental value. Similarly, the measured resonance energies lie about $2 \mathrm{eV}$ below our theoretical values. We attribute these differences to missing correlation effects in the calculations. This is not surprising because the configuration interaction method, which is widely used by the community, converges very slowly for neutral atoms, making it very difficult for theory to obtain good agreement with experiment, as we discuss in more detail below. Another noticeable difference is the strength of the $1 s 2 s^{2} 2 p^{3} 3 p$ resonance, which is higher in our calculations, and the energy, which is lower. We should again note that the spurious 
molecular contribution mentioned above, possibly contaminating the atomic nitrogen experimental results, may play a role in the discrepancies between experimental and theoretical results. Additionally, it is interesting that current calculations for cross sections are in better agreement with the measurements of Henke et al. (1993). We attribute this to the lack of correlation effects in both works.

Overall, better agreement between theory and experiment for the TIY cross section was found for the R-matrix calculations of Sant'Anna et al. (2011). Still, we do find good agreement between the R-matrix results and the present calculations for the background cross sections. For example, the previous calculations give about $0.8 \mathrm{Mb}$ at $415 \mathrm{eV}$, while our value is $0.7 \mathrm{Mb}$ at that energy. On the other hand, our approach has been successfully used for the analysis of branching ratios in inner-shell ionization of krypton and xenon atoms (Jonauskas et al. 2003, 2011; Palaudoux et al. 2010). In principle, it should be possible to perform R-matrix calculations for branching ratios too. However, we are unaware of any such published studies.

Of particular interest is the baseline behavior in the $\mathrm{N}^{+}$and $\mathrm{N}^{2+}$ branching ratios from threshold to about $435 \mathrm{eV}$. Over this range, the experimental $\mathrm{N}^{+}$branching ratio starts above theory, but then decreases into good agreement, while the $\mathrm{N}^{2+}$ measurements start below theory, but then increases into good agreement. This behavior is not predicted by our theory and the cause for the discrepancy is unclear. It is unlikely to be due to post-collision interactions (PCIs), which can occur just above a photoionization threshold. Such interactions could prevent a slow photoelectron from actually escaping from the system, thus reducing the $\mathrm{N}^{2+}$ yield and enhancing the $\mathrm{N}^{+}$yield for the first few eV above an ionization threshold. However, PCI is predicted not to be strong enough to account for the magnitude of the effect seen. Any variation in the partial cross section for L-shell photoionization is also unlikely to be the cause. We are over an order of magnitude in energy above the L-shell ionization threshold and the L-shell partial cross section is predicted to be smooth in this energy range. One possible explanation for the differences could be missing correlation effects for the excited configurations. A much larger basis set of interacting configurations would lead to a larger contribution to the cross section from photoexcitiation. However, the basis of interacting configurations must also include mixing with continua as well. Our initial estimates show that a computationally prohibitive basis set would be required, just for the bound configurations. An additional difference we see between our experimental and theoretical results is the apparent anticorrelation between the peaks and the valleys in the structure of the branching ratios. These findings imply that direct double Auger or even triple Auger transitions may be involved in the formation of the doubly and triply ionized stages.

The series limit is near $444 \mathrm{eV}$. The resonance can be seen in the total cross section, in the $\mathrm{N}^{3+}$ branching ratio, to a lesser extent in the $\mathrm{N}^{2+}$ data, and by a dip in the $\mathrm{N}^{+}$results. Unfortunately, due to the small cross section and the measurement noise levels, we are unable to perform a Rydberg analysis to experimentally determine these ionization thresholds.

For $\mathrm{N}^{2+}$ and $\mathrm{N}^{3+}$, we find good agreement between our experimental and theoretical results above $435 \mathrm{eV}$. This demonstrates that DPH can be successfully described as arising from two sequential processes. On the other hand, shake-off calculations, which are not included in the results here, are higher than the experimental results by about a factor of two at $470 \mathrm{eV}$.

Looking at all three final charge states produced, we see that eventually the branching ratios and final average charge state all appear to reach asymptotic values at about $470 \mathrm{eV}$. The only processes that are possible at higher energies, are double $\mathrm{K}$-shell ionization and direct triple-ionization $(\mathrm{K}+\mathrm{L}+\mathrm{L})$, both of which are expected to be negligibly small.

Comparing to the results of Kaastra \& Mewe (1993), we find significant differences in the branching ratios and average final charge state. Given the theoretical calculations that their work is derived from, it is not surprising that they do not include any of the observed resonance structure and energy dependence. These differences remain even where the experimental results appear to have reached their asymptotic limit. Our measured branching ratios are larger than Kaastra \& Mewe (1993) for forming $\mathrm{N}^{+}$and smaller for forming $\mathrm{N}^{2+}$. Moreover, we find a significant branching ratio for forming $\mathrm{N}^{3+}$, a channel that was considered to be closed by Kaastra \& Mewe (1993). Thus it appears that K-shell ionization processes can increase the average charge state of a cosmic plasma faster than is currently predicted by models. Exploring the full astrophysical implications of our experimental findings, which will require implementing our data into various astrophysical models, is beyond the scope of our work here.

\section{SUMMARY}

We have reported inner-shell photoionization measurements and calculations of atomic nitrogen near the $1 s$-ionization threshold. Significant differences are found with the recommended data currently used by the astrophysics community, particularly for the final CSD. For example, the branching ratio for $\mathrm{N}^{+}$formation is much greater than that given in the data base, as well as that for $\mathrm{N}^{3+}$, a channel that the recommended data predict is closed. These results point out some of the shortcomings in the 40-year-old theoretical data currently available for the CSD due to inner-shell ionization. Generating the state-of-the-art data necessary in order to produce more reliable modeling of cosmic sources will require a concerted theoretical and experimental effort covering all systems where a $1 s$-hole can be formed. The present theoretical work is an initial attempt at calculating atomic nitrogen branching ratios for K-shell photoionization using the FAC method. The poor agreement with the experimental results suggests that a more sophisticated theoretical treatment is required.

We dedicate this work to the memory of our dear colleague and co-author Dennis Lindle, who passed away during the writing of this paper. The authors thank J. Kaastra, T. R. Kallman, R. K. Smith, and E. M. Gullikson for stimulating conversations. W.C.S. and D.W.L. wish to acknowledge support by the National Science Foundation under NSF Grant No. PHY-09-70125. V.J. was funded by the European Social Fund under the Global Grant Measure (No. VP1-3.1-ŠMM-07-K-02015). M.M.S. was supported by the CNPq-Brazil. D.W.S. was supported in part by the NASA Astrophysics Research and Analysis Program and the NASA Heliophysics Supporting Research Program. The experimental portion of this work was performed at the Advanced Light Source, which is supported by DOE (DE-AC03-76SF00098). 


\section{REFERENCES}

Bautista, M. A., Mendoza, C., Kallman, T. R., \& Palmeri, P. 2003, A\&A, 403, 339

Chen, C. T., Ma, Y., \& Sette, F. 1989, PhRvA, 40, 6737

Cowan, R. D. 1981, The Theory of Atomic Structure and Spectra (Berkeley, CA: Univ. California Press)

Dwek, E., \& Smith, R. K. 1996, ApJ, 459, 686

García, J., Kallman, T. R., Witthoeft, M., et al. 2009, ApJS, 185, 477

García, J., Mendoza, C., Bautista, M. A., et al. 2005, ApJS, 158, 68

Gorczyca, T. W., Dumitriu, I., Hasoğlu, M. F., et al. 2006, ApJL, 638, L121

Gorczyca, T. W., Kodituwakku, C. N., Korista, K. T., et al. 2003, ApJ, 592 636

Gryziński, M. 1965, PhRv, 138, A336

Gu, M. F. 2008, CaJPh, 86, 675

Hahn, M., \& Savin, D. W. 2015a, ApJ, 809, 178

Hahn, M., \& Savin, D. W. 2015b, ApJ, 800, 68

Hasoğlu, M. F., Gorczyca, T. W., Korista, K. T., et al. 2006, ApJL, 649, L149

Henke, B. L., Gullikson, E. M., \& Davis, J. C. 1993, ADNDT, 54, 181

Jonauskas, V., Kučas, S., \& Karazija, R. 2009, Lith. J. Phys., 49, 415

Jonauskas, V., Kučas, S., \& Karazija, R. 2011, PhRvA, 84, 53415

Jonauskas, V., Partanen, L., Kučas, S., et al. 2003, JPhB, 36, 4403

Jonauskas, V., Prancikevičius, A., Masys, Š., \& Kynienè, A. 2014, PhRvA, 89, 052714

Kaastra, J. S., \& Mewe, R. 1993, A\&AS, 97, 443

Kallman, T., \& Bautista, M. 2001, ApJS, 133, 221
McLaughlin, B. M., Ballance, C. P., Bowen, K. P., Gardenghi, D. J., \& Stolte, W. C. 2013, ApJL, 771, L8

Mendoza, C., Kallman, T. R., Bautista, M. A., \& Palmeri, P. 2004, A\&A, 414, 377

Palaudoux, J., Lablanquie, P., Andric, L., et al. 2010, PhRvA, 82, 43419

Palmeri, P., Mendoza, C., Kallman, T. R., \& Bautista, M. A. 2003a, A\&A, 403, 1175

Palmeri, P., Mendoza, C., Kallman, T. R., Bautista, M. A., \& Meléndez, M. 2003b, A\&A, 410, 359

Palmeri, P., Quinet, P., Mendoza, C., et al. 2008a, ApJS, 177, 408

Palmeri, P., Quinet, P., Mendoza, C., et al. 2008b, ApJS, 179, 542

Palmeri, P., Quinet, P., Mendoza, C., et al. 2011, A\&A, 525, A59

Palmeri, P., Quinet, P., Mendoza, C., et al. 2012, A\&A, 543, A44

Samson, J. A. R., \& Angel, G. C. 1990, PhRvA, 42, 1307

Samson, J. A. R., \& Pareek, P. N. 1985, PhRvA, 31, 1470

Samson, J. A. R., Shefer, Y., \& Angel, G. C. 1986, PhRvL, 56, 2020

Sant'Anna, M. M., Schlachter, A. S., Öhrwall, G., et al. 2011, PhRvL, 107, 33001

Semenov, S. K., Cherepkov, N. A., Matsumoto, M., et al. 2006, JPhB, 39, 375

Stolte, W. C., Felfli, Z. G. R., Öhrwall, G., et al. 2013, PhRvA, 88, 53425

Stolte, W. C., Guillemin, R., Yu, S.-W., \& Lindle, D. W. 2008, JPhB, 41, 145102

Stolte, W. C., He, Z. X., Cutler, J. N., Lu, Y., \& Samson, J. A. R. 1998, ADNDT, 69, 171

Stolte, W. C., Lu, Y., Samson, J. A. R., et al. 1997, JPhB, 30, 4489

Vink, J. 2012, ARA\&A, 20, 49 